\title{
Exceptuando normas: apuntes para un análisis conceptual
}

\section{Excepting Norms: Notes for a Conceptual Analysis}

\author{
Víctor García Yzaguirre
}

\author{
Autor: \\ Víctor García Yzaguirre \\ Universidad Austral de Chile \\ Universidad de Génova, Italia \\ garciayzaguirre@gmail.com \\ https://orcid.org/0000-0002-4662-2919 \\ Recibido: 13-5-2019 \\ Aceptado: 4-1-2020

\section{Citar como:} \\ García Yzaguirre, Víctor, (2020). Exceptuando \\ normas: apuntes para un análisis conceptual. \\ Doxa. Cuadernos de Filosofía del Derecho, \\ 43, pp. 427-456. https://doi.org/10.14198/ \\ DOXA2020.43.16

\section{Licencia:} \\ Este trabajo se publica bajo una Licencia Creative \\ Commons Atribución 4.0 Internacional.

\section{(c) (i)}

(C) Víctor García Yzaguirre

\begin{abstract}
Resumen
El objeto del presente trabajo es analizar el contenido conceptual de la noción de excepción. Para ello, describiré y analizaré algunas de sus principales conceptualizaciones formuladas desde la teoría del derecho, presentándolas como una discusión entre teóricos del derecho que consideran que las excepciones únicamente dan cuenta de una descripción retórica de los antecedentes de las normas frente a quienes sostienes que esta posee contenido conceptual. Concluiré que la mejor forma de entender qué es exceptuar una norma es como un concepto que permite esclarecer los actos mediante los cuales se reduce el ámbito de aplicación de las normas.
\end{abstract}

Palabras clave: excepciones; norma; estructura de normas

\begin{abstract}
The purpose of this paper is to analyze the conceptual content of the notion of exception. To do this, I will describe and analyze some of its main conceptualizations formulated from the theory of law, presenting them as a discussion among law theorists who believe that the exceptions only account for a rhetorical description of the background of the norms against those who hold that It has conceptual content. I will conclude that the best way to understand what is to except a norm is as a concept that allows to clarify the acts by which the scope of the norms is reduced.
\end{abstract}

Keywords: exceptions; norms; structure of norms

\footnotetext{
* Este trabajo se enmarca como tesista del proyecto Fondecyt «Hacia una teoría del stare decisis» (núm. 1180494) concedido por el Conicyt chileno. Agradezco a Álvaro Núńez Vaquero, Leticia Morales, Omar Sar Suárez, José Fernández Cruz, Carla Iuspa Santelices, Pablo Durand Leiva, Patricio Rondini Fernández-Dávila, y a los dos árbitros anónimos de este texto por sus atentas y agudas observaciones, muchas de las cuales sigo adeudando resolver.
} 


\section{INTRODUCCIÓN}

|

maginemos que estamos caminando por un parque y nos topamos con una señal municipal que dice "prohibido aparcar vehículos, salvo ambulancias». En ese momento nuestro acompañante, quien es un teórico del derecho, nos dice "“ambulancia” es la excepción a la norma», ¿qué nos está diciendo con ello?

Julius STONe, en una de sus obras más difundidas, sostuvo que el concepto de excepción era una categoría con referencia ilusoria por carecer de significado. Ello, debido a que consideró que quienes hacen una diferencia entre un requisito de aplicabilidad y una excepción a una norma están realizando una distinción entre objetos que no son diferenciables. No habría, según él, ninguna diferencia entre señalar «si algo es $\mathrm{X}$ y no $\mathrm{Y}$, entonces es Z» y «si algo es X, entonces es Z, a menos que Y». Predicar que una norma está exceptuada, en este escenario, solo daría cuenta de un uso retórico sobre cómo describimos el antecedente de una norma ${ }^{1}$. Al respecto, predicar que una propiedad es una excepción, ¿esclarece algún aspecto teórico relevante?

Numerosos teóricos del derecho han propuesto y discutido sobre las diversas conceptualizaciones de la noción de norma, pero no han sido muchos quienes se han propuesto hacer lo mismo respecto a la noción de excepción. Es un problema relevante, pues mediante un adecuado concepto de excepción podemos mejorar tanto nuestras técnicas legislativas como nuestras prácticas de aplicación de normas.

El objetivo del presente trabajo es dar razones para justificar que la noción de excepción nos permite esclarecer aspectos teóricos relevantes sobre la estructura de las normas y las operaciones que realizamos con normas. En este sentido, voy a sostener que la mejor manera de presentar esta noción es formulándola como un verbo, «exceptuar», la cual da cuenta de cómo se introducen nuevas propiedades en una norma con el efecto de reducir el alcance del caso genérico previsto en el antecedente. Para ello, analizaré y criticaré algunas de las principales propuestas de conceptualización de excepción disponibles en la teoría del derecho. En relación a este último punto, cabe precisar que, si bien este es un concepto empleado en todas las áreas del derecho, solo analizaré propuestas de teóricos del derecho ${ }^{2}$.

1. Lo mismo sucede en los casos que los aplicadores del derecho separan entre normas que incluyen una excepción de normas que exceptúan a otras. STONE argumentó cómo esta distinción, creada a partir de la idea de identificación, era solo una manera de encubrir la creación de reglas en materia de inversión de la carga de la prueba sin confesarlo o ser transparente en ello. STONE, 1985: 68-71. Cabe anotar que BeNTHAM ya había formulado una advertencia similar. Él propuso contrastar entre «nadie puede exportar trigo si el precio de mercado excede los 44 chelines por libra» con «se puede exportar trigo, a menos que el precio de mercado exceda los 44 chelines por libra». La diferencia entre un limitante y una excepción solo sería una formalidad. Bentham, 1970: 114. Sobre este punto ver Raz, 1986: 78 y ss.

2. Debo precisar que las reconstrucciones de los autores citados serán parciales, en tanto solo analizaré los extremos relevantes al objeto del presente trabajo. Asimismo, no me ocuparé con precisión de la distinción entre excepciones explícitas e implícitas, pues me preocupa aclarar, en este momento, qué ha sido propuesto para entender los casos claros de excepción explícita de las normas. En relación a las propuestas dogmáticas de clarificación de la noción de excepción, su estudio es una tarea pendiente. 
A afectos de presentar las diversas propuestas de conceptualización, las voy a ordenar en dos conjuntos: i) aproximación retórica (agrupa a quienes consideran que la noción de excepción esclarece, únicamente, un uso retórico de nuestras descripciones de las normas); y ii) aproximación conceptualista (agrupa a quienes consideran que la noción de excepción esclarece aspectos normativos relevantes de las normas) ${ }^{3}$. Tras ello analizaré brevemente, asumiendo una postura conceptualista, las implicancias de renunciar a la posibilidad de exceptuar normas jurídicas.

\section{EXCEPCIÓN: ¿TIPO DE NORMA O PARTE ESTRUCTURAL DE LAS NORMAS?}

Previo a analizar las propuestas teóricas sobre qué es una excepción (como problema conceptual), es pertinente clarificar qué tipo de problema es identificar una excepción. Entiendo esta pregunta como la duda respecto a si una excepción es un tipo de norma o si dicha noción se emplea para dar cuenta de los componentes de una norma ya identificada.

El análisis conceptual sobre qué son las excepciones está vinculado con diversas discusiones abiertas de teoría del derecho. En particular, es relevante su conexión con los problemas de individualización y estructura de las normas. Los primeros refieren a las discusiones teóricas sobre cómo descomponer el material jurídico de manera de presentar el derecho en unidades que sean calificables de normas jurídicas ${ }^{4}$. La segunda, en cambio, se refiere a las discusiones teóricas sobre cómo están estructuradas las normas identificadas (qué elementos las conforman, cómo están articulados estos elementos, qué implican estos elementos). Ambos temas son interdependientes, pero pretenden responder a preguntas distintas.

Parto por asumir que no existe una forma teórica de exponer la estructura, identidad o individualidad de las normas que sea correcta, en abstracto, por sobre las demás. De lo que disponemos son nociones empleadas para analizar el derecho desde un plano teórico con distintos propósitos. Suponer lo contrario es, como bien señala Honoré, convertirse en víctima de una extraña forma de metafísica analíticas

3. Cabe indicar que en la literatura son identificables quienes siguen una aproximación no deductivista de las normas y de las excepciones. No daré cuenta de estos autores, pues se requeriría una exploración de las razones por las cuales se abandona la deducibilidad como medio para generar conclusiones, lo cual excedería nuestros propósitos. Una aproximación diferente a esta problemática puede verse en ANDRADE, 2018.

4. BAYÓN, 2003: 290.

5. Honore, 1977, p. 100. Dicho autor ha resaltado el problema de la individualización a partir del uso profesional que realizan los estudiosos del derecho del término norma. En efecto, no se realiza una equivalencia entre un acto de promulgación y una norma, no es lo mismo, necesariamente, una regla que un artículo de una ley. $\mathrm{Ni}$ tampoco lo es una regla jurisprudencialmente creada con una sentencia. Lo que hacemos es extraer reglas, no mediante actos de reproducción, sino por actos de individualización empleando material jurídico que puede ser diverso. HONORE, 1977: 100-101. 
La individualización y la estructuración de las normas refieren a preguntas teóricas diferentes, pero vinculadas estrechamente, pues las respuestas que demos a la segunda esclarecen de los elementos de la primera. Las discusiones sobre la individualización de normas parten de preguntarse cuáles son los criterios que hemos de emplear para determinar qué normas hay dentro de un sistema normativo. En otros términos, engloba las propuestas y debates sobre qué criterios se deben emplear para determinar la identidad de las normas (qué es una norma, qué es un fragmento de norma, qué no es una norma, entre otras $)^{6}$.

Las discusiones sobre la estructura de normas, si bien dependen ${ }^{7}$ de la respuesta que se dé a la pregunta sobre cómo identificar una norma ${ }^{8}$, poseen temáticas propias.

Engloban las propuestas sobre cómo representar una norma, en el sentido de determinar cuáles son sus componentes y cómo se articulan.

Siguiendo a Richard Susskind, la pregunta sobre la estructura busca respuestas de tipo sintáctico, es decir, son discusiones abocadas a determinar qué elementos componen una norma con independencia de su contenido. En cambio, las discusiones sobre la individualización tienen como objetivo establecer el contenido semántico de los elementos constitutivos de las normas. Se refiere, en este sentido, a los criterios que empleamos para dividir el material jurídico en normas, de manera coherente con una teoría sobre qué son las normas?.

Cabe precisar que el contenido de las discusiones sobre la individualización como de la estructuración de una norma está determinado por el tipo de análisis que hagamos del derecho. En efecto, la elección de una propuesta respecto a los criterios a emplear para identificar normas o para representarlas depende de qué se considere relevante de esclarecer. En cambio, el debate sobre estructura de normas condicionales se ha caracterizado por dos posturas en competición: la concepción puente y la concepción insular de normas ${ }^{10}$. Dados los objetivos del presente documento no profundizaré sobre las diferencias en cada una de estas discusiones ${ }^{11}$.

6. RAZ, 1986: 97, HART, 1982: 107.

7. Depende de dos formas: i) se discutirá sobre la estructura siempre que tengamos normas identificadas; y ii) la discusión sobre cómo están estructuradas las normas está condicionada a los criterios de identificación de normas, por cuanto ello determinará qué tipo de información (y cuanta información) se va presentar de forma estructurada.

8. Siguiendo el ejemplo de Raz, podemos tener tres normas con una composición idéntica (pensemos que cada una de estas regula a un tipo de sujeto diferente, pero comparten todas la misma acción y la misma calificación deóntica) y una cuarta que las agrupa todas en contenido. Todas poseen la misma estructura, pero solo la cuarta es la que describe por completo la norma que pertenece al sistema normativo Raz, 1986: 99.

9. SussKind, 1987: 119.

10. Rodríguez, 2005.

11. Adoptaré en este trabajo la concepción puente las normas condicionales representadas de la siguiente forma: (p->Oq). De esta forma el operador deóntico solo afecta el consecuente. Esto quiere decir que el compromiso deóntico asumido bajo esta propuesta es que las normas condicionales son un puente que vincula lo que es (o podría ser) un caso con lo que debe ser. Asimismo, asumiré que las normas validan tanto el refuerzo del antecedente como la regla del modus ponens. Al respecto ver Alchourrón, 2010, p. 144. ZuLETA, 2008, p. 99.

Doxa. Cuadernos de Filosofía del Derecho, (2020), 43, pp. 427-456 
Las categorías propuestas sobre la estructura de normas pueden ser, a su vez, profundizadas realizado distinciones y subdistinciones entre componentes estructurales y elementos que conforman cada componente. Por ejemplo, Richard Susskind ${ }^{12}$ ha formulado una propuesta de componentes de los antecedentes normativos dentro de la concepción puente de las normas. De acuerdo con esta concepción, la estructura general de las normas tiene dos componentes estructurales que forman el enunciado condicional «Si p, entonces q", entendiendo por $\mathrm{p}$ el antecedente y por q el consecuente. Dentro de cada uno de estos elementos estructurales podemos identificar "compartimentos»" ${ }^{13}$ o sub elementos estructurales, de los cuales solo nos interesan (para los efectos del presente trabajo), aquellos contenidos en el antecedente. Cada uno de estos compartimentos da cuenta del tipo de condición o condiciones con los cuales se ha construido el antecedente.

Los compartimentos del antecedente, conforme a esta propuesta, nos permiten representar cuatro tipos de condiciones: i) condición principal, ii) condición alternativa, iii) condición conjuntiva; y iv) condición de excepción ${ }^{14}$. Cada una de estas condiciones puede referir a actos, acciones, eventos o situaciones, es decir, a hechos brutos o institucionales. Esta referencia puede ser, bien positiva (se realizó una acción u ocurrió un hecho), bien negativa (no se realizó una acción o no ocurrió un hecho).

Las nociones de condición principal, alternativa y la conjuntiva de aplicación dan cuenta de todos los hechos o acciones que, de ser verificados en un caso individual, hacen que entonces sea aplicable una norma jurídica (con todas aquellas propiedades que refieran a las acciones y/o hechos que se pretenden regular). En cambio, la noción de condición de excepción refiere a todos los hechos o acciones que, de ser verificados en un caso individual, hace que no sea aplicable la norma ${ }^{15}$, en tanto, estarían fuera

12. SusSKIND, 1987, p. 130.

13. Susskind empleó el término «tolken» el cual traduzco, a falta de una propuesta más precisa y elegante, como compartimento.

14. SussKIND, 1987: 133. Un antecedente compuesto por una condición principal de aplicación refiere a que se sigue el consecuente de verificarse un hecho o acción (p); una condición alternativa de aplicación refiere a que se sigue el consecuente de verificarse dos hechos o acciones, se den conjuntamente o por lo menos uno de estos (pvq); una condición conjuntiva de aplicación refiere a que se sigue el consecuente de verificarse dos hechos o acciones conjuntamente (p.q); y una condición de excepción de aplicación refiere a que no se sigue el consecuente de verificarse dos hechos o acciones conjuntamente (p.q). Como vemos, el problema está como distinguir entre estas dos últimas condiciones de aplicación.

15. Susskind en este punto sigue la distinción propuesta en HoHFeLd, 1991: 43. Dicho autor, para ejemplificar su distinción, no propone pensar en los hechos operativos de un contrato entre A y B. Los afirmativos serían que cada parte sea humana, tenga por lo menos una determinada edad, que una haya ofrecido, que la otra haya aceptado la oferta, entre otros. Los negativos serían que A haya inducido dolosamente a error a B o que A no haya revocado su oferta. Precisamos que la referencia «afirmativa» $\mathrm{y}$ "negativa» de los hechos operativos no refiere a si el hecho debe ser positivo (de hacer o existencia) o negativo (de omitir o ausencia), pues tanto el afirmativo como el negativo podrían estar compuestos por cualquier hecho positivo o negativo o una combinación de estos. 
de su alcance ${ }^{16}$. Pero esto solo nos devuelve al problema que nos propuso STONE: ¿hay diferencia entre estas condiciones o es solo retórica?

Para ilustrar este punto voy a seguir un ejemplo propuesto por NeIL MACCormick ${ }^{17}$ : una persona contrata un barco para el transporte de mercancías, y en su travesía la embarcación se hunde, de lo que se sigue que no puede entregar las mercancías conforme a lo pactado en el contrato. $\mathrm{Al}$ respecto, cabe anotar que la regulación vigente para el caso establece que en supuestos de incumplimiento sin culpa (o frustración no autoinducida) del contrato, no se genera responsabilidad civil.

¿Cómo podríamos representar la norma que regula la responsabilidad civil de contratos incumplidos? Consideremos el siguiente enunciado: «el contratista que incumple su contrato tiene la obligación de pagar una indemnización». Formalmente esto se representa así:

$$
(\mathrm{p} \rightarrow \mathrm{Oi})
$$

La p representa el hecho de que una persona que contrató con otra incumplió con sus deberes contractuales ${ }^{18}$. La Oi representa la obligación de pagar la indemnización. Esta representación, como vemos, está incompleta. Para que se siga el consecuente tiene que ser el caso que no se haya dado una frustración autoinducida ${ }^{19}$. Esto nos lleva a la siguiente representación.

$$
((\mathrm{p} . \neg \mathrm{r}) \rightarrow \mathrm{Oi}))
$$

La $\mathrm{r}$ representa el hecho de que la frustración del contrato haya sido autoinducida. El operador $(\neg)$ representa la negación, por lo que $\neg$ r quiere dar cuenta de que no hubo frustración de contrato autoinducida. En este punto cabe resaltar que las propiedades del antecedente (p. $\neg$ r) operan como conjunción, es decir, se deben dar ambas en el caso para que se siga el consecuente.

16. Como bien han señalado Moreso y Navarro (Moreso y Navarro, 1996) el término «aplicabilidad» es ambiguo, pues puede ser entendido como aplicabilidad interna (o alcance), o como aplicabilidad externa (o fuerza). En este sentido, una norma es internamente aplicable a un caso particular siempre que este sea una instanciación o ejemplificación del caso genérico contenido en el antecedente de la norma. En cambio, una norma es externamente aplicable a un caso individual siempre se satisfagan conjuntamente las siguientes condiciones: i) sea internamente aplicable; y ii) otra norma N2, perteneciente al sistema jurídico, ordene al aplicador del derecho a emplear N1 en la justificación de sus decisiones cada vez que estén frente a una ejemplificación del caso genérico C. En adelante al hablar de aplicabilidad la entenderé como aplicabilidad interna.

17. МacСormick, 2016, p. 405, МacСormick, 1995: 104. Se inspiró en el caso Joseph Constantine Steamship Line Ltd. v. Imperial Smelting resuelto por la House of Lords Appeal Committee del Reino Unido.

18. Otra forma de representar esta proposición es ( $\neg p->\mathrm{Oi})$ donde $\mathrm{p}$ representa el hecho de que una persona que contrató con otra cumplió con sus deberes contractuales, de manera que al negar esto con el operador ( $\neg$ ) estamos diciendo que la persona no cumplió con sus deberes contractuales. Dado que el punto a esclarecer en este momento es cómo representar una propiedad que opera como excepción, me ha parecido que genera mayor claridad que no todas las propiedades del antecedente se representen como negativos.

19. Otra forma de representar «no se haya dado una frustración autoinducida» es señalar "culpa», pero esto no me permitiría hacer más clara la idea a explicitar mediante la formalización. 
En la representación $((\mathrm{p} . \neg \mathrm{r}) \rightarrow \mathrm{Oi}))$ no es posible identificar condición de excepción del resto de condiciones, pues tanto la p como la $\neg$ r operan en conjunción. En el lenguaje natural una excepción podría ser presentada empleando un "pero» o un "a menos que» a efectos de introducir una conjunción adversativa entre dos elementos oracionales. Ello no puede ser capturado por la conjunción lógica la cual, en el lenguaje natural, da cuenta en su versión incluyente de la «y» (como conjunción copulativa) y en su versión excluyente de la «o» (como conjunción disyuntiva). En un antecedente, de esta forma, desde el plano lógico un "pero» es tratado de la misma forma en que se trata una "y»" ${ }^{20}$. En este sentido, la distinción entre una excepción y el resto de condiciones es algo de lo que no podemos dar cuenta desde una aproximación lógica ${ }^{21}$.

Este ejemplo nos pone de relieve otro aspecto a clarificar: la $\neg \mathrm{r}$ (asumamos, por el momento, que esta es una excepción), no tiene el efecto de negar las otras propiedades. La diferencia entre negar una propiedad de exceptuar una norma es la misma que hay entre "yo no lo hice» con "lo hice, pero...», la primera implica negar un hecho, la segunda, en cambio, refiere a aceptar el hecho, pero que este es concurrente con otros hechos que le exoneran de responsabilidad (o, en términos más abstractos, hacen inaplicable la consecuencia normativa).

Este efecto inaplicador puede ser esclarecido si consideramos la idea de "palabras excluidoras» de Roland HalL ${ }^{22}$, quien a su vez desarrolla la distinción de J. L. Austin entre palabras usadas para negar y usadas para oponer ${ }^{23}$. Hall está dando cuenta de los usos habituales de palabras que informan a manera de descarte, por ejemplo, «real», «civil» o «bárbaro». Estas son usadas, principalmente, de manera negativa, es decir, para excluir caracterizaciones, modos o manifestaciones ajenos a los que se pretende aludir. Estas no niegan la existencia de un hecho, sino que se usan para excluir la aplicabilidad de ciertas propiedades en determinados contextos. Conforme a los ejemplos, «civil»se emplearía para excluir a eclesiástico o militar, «real» para excluir a imaginario o figurativo («tigre real», por ejemplo, informa que no estamos ante un tigre de juguete o el dibujo de un tigre) y «bárbaro» era empleado en tiempos clásicos para hablar de no griegos y, posteriormente, no romanos. En breve, excluidor refiere al uso de una palabra no para denotar una propiedad positiva, sino para excluir propiedades y contextos de uso.

20. MacCormick, 2016: 407. Cabe anotar que «la práctica de la diferente asignación de cargas de la prueba no puede estar exigida lógicamente por la supuesta diferencia en los tipos diferentes de condición jurídica. Pues no difieren lógicamente». MaCCormick, 2016: 406. Sin perjuicio de lo anotado, la lógica si nos permite dar cuenta del tipo de actividades que hemos realizado para incorporar la $\neg \mathrm{r}$ en el antecedente, es decir, si nos podría esclarecer lo que hacemos al momento de exceptuar una norma. Pero sobre esta idea volveré más adelante.

21. Cabe resaltar que otra forma de enfrentar esta situación es cambiando de lógica, pero sobre este punto no profundizaré. A efectos de un ejemplo ver Prakken, 1997: 33 y ss.

22. Hall señala que son palabras caracterizadas por: i) atribuir opuestos; ii) descartar algo sin agregar ellas mismas información adicional sobre el objetivo; y iii) reducen el grado de ambigüedad contextual (descartan contextos de uso del término). Hall, 1959: 1.

23. Austin, 1975: 181, Austin, 1981: 98. 
La concurrencia de las excepciones con el resto de propiedades prevista en el antecedente opera de manera análoga a este uso exclusorio de las palabras: su uso no niega la existencia de la ocurrencia de un hecho, sino que se opone a que se aplique un determinado predicado ${ }^{24}$. No dan cuenta de la ausencia de hechos, sino que refieren a que un hecho $\mathrm{H} 1$ sucedió de manera concurrente con un hecho $\mathrm{H} 2$, y que el efecto de haber verificado $\mathrm{H} 2$ es de oponerse a que se pueda hacer inferencias prácticas de haber verificado $\mathrm{H} 1^{25}$.

Esta conclusión nos devuelve a la propuesta estructuralista de Susskind la cual nos sirve para poner de relieve algunos puntos problemáticos. Esta propuesta tiene el acierto de proponernos un esquema de clasificación a emplear sobre condiciones del antecedente, el problema es que no ofrece ni reenvía a ninguna propuesta de criterios de identificación de estas condiciones. Susskind ha formulado sus tesis a partir de un análisis lógico-formal que señala, sin demostrar que hay diferencias entre una condición de aplicación conjuntiva y una de excepción. En este sentido, esta propuesta es una invitación a preguntarnos: ¿cómo podríamos identificar una condición de excepción? Si no es posible identificarla mediante un análisis lógico, ¿qué tipo de análisis debemos realizar?

\section{EXCEPCIONES: ENTRE USOS RETÓRICOS Y PROPUESTAS CONCEPTUALES}

La discusión teórica sobre la identificación de condiciones de excepción se ha caracterizado por el antagonismo entre dos posiciones: por un lado, quienes consideran que el uso del término «excepción» es solo una forma de hablar, pues exceptuar no es otra cosa que no aplicar una norma por estar fuera de su alcance; por el otro, quienes consideran que el término «excepción» refiere a conceptos que nos permiten esclarecer rasgos distintivos de algunas condiciones del antecedente. Veamos algunas de estas propuestas:

24. En relación a este efecto exclusorio es pertinente poner de relieve, a efectos de precisión, la diferencia entre justificación y excusa de la acción. Por justificación de la acción, siguiendo a Austin, entiendo los actos de aceptación responsabilidad por un acto, pero negamos que esta sea contraria al sistema normativo. En cambio, excusa refiere a la aceptación de que se cometió una acción contraria al sistema normativo, pero que esta es concurrente con otras acciones o eventos que exoneran de responsabilidad. Austin, 1975: 172. Esta distinción se aclara si consideramos el rol del afectado: si un acto está excusado, entonces la víctima tuvo todo el derecho de resistirse al daño. En cambio, si un acto está justificado, entonces no hay víctima, pues la persona afectada no tuvo derecho a resistirse al acto. Hablar de efecto exclusorio de las excepciones, conforme a estas nociones, es hablar de excusas. Cabe indicar que la distinción entre justificación y excusa no ha estado libre de discusión, por ejemplo: Greenawalt, 1986.

25. Cabe insistir que estamos en el plano de usos del lenguaje. La noción de palabra exclusoria refiere a su uso, no a su contenido semántico. Sin embargo, como bien señala HALL, podemos emplearla como definición o explicación de un objeto, pero esa aproximación no capturaría eso que pretendemos hacer cuando la usamos. Hall, 1959: 5. 


\section{a. Aproximación retórica}

De acuerdo con este primer conjunto de propuestas las excepciones son reducibles a elementos en el antecedente que especifican las propiedades que deben verificarse para aplicar la norma. En este sentido, aquí se agrupan a todos aquellos que sostienen que el término "excepción" es solo un recurso retórico explicativo para dar cuenta de los antecedentes, pero sin contenido conceptual propio. Al respecto, paso a reconstruir las propuestas de Glanvile Williams y Frederick Schauer al ser claros ejemplos de este conjunto.

\section{i. Excepciones como delimitaciones del antecedente}

Glanville Williams, en un célebre artículo ${ }^{26}$, nos propone contrastar dos casos. Para el primero pensemos en el caso de una persona que tiene acceso carnal con otra sin su consentimiento, por lo que la fiscalía exige la aplicación de la norma que prevé el delito de violación sexual. Para el segundo imaginemos a una persona que agrede físicamente a otra, ante lo cual la agredida requiere la aplicación de la norma que prevé el delito de lesiones.

En el primer caso la fiscalía debe demostrar la ausencia de consentimiento. En el segundo, la pretensión se sostiene, explícita o implícitamente, sobre la premisa de que la agresión sucedió sin la concurrencia de una causa de justificación, como podría ser la legítima defensa. ¿Estos casos son normativamente diferentes?

En el segundo caso una de las maneras del acusado para no padecer las consecuencias jurídicas del delito de lesiones es demostrar la aplicabilidad de la legítima defensa, pues su efecto conllevaría la no aplicación del consecuente (sanción penal). De esta forma, la legítima defensa, comenta Williams, parecería una excepción. Ahora bien, ¿acaso esta no opera como un elemento negativo de la norma, como lo es la ausencia de consentimiento en el caso de violación? ${ }^{27}$ Una lesión, de esta forma, no es otra cosa que una agresión física a otra persona no motivada por la legítima defensa.

La acusación por violación es por sexo sin consentimiento; la acusación por lesión se basa en una agresión física. Pero esta última no es cualquier acción agresiva, sino que cuenta aquella efectuada de manera ilegal. De esta forma, el elemento «ilegal» es una propiedad integrante de la definición de lesión, es decir, implica que la persona acusada no actuó conforme a ninguna causal eximente de responsabilidad. La legítima defensa, entonces, se explica como una condición necesaria (y negativa, es decir no ocurrió una hecho o acción) del antecedente de la norma (en este caso, de la norma que prevé un delito) ${ }^{28}$. Dicho en breve, a partir de esta aproximación de Williams,

26. Williams, 1988: 276-77.

27. Williams, 1988: 279.

28. Williams, 1988: 279.

Doxa. Cuadernos de Filosofía del Derecho, (2020), 43, pp. 427-456 
las excepciones no son otra cosa que delimitadores (o especificaciones) del ámbito de aplicación de las normas ${ }^{29}$.

Williams sostuvo que buscar la línea que divide la excepción del resto de condicionales es como buscar en un cuarto oscuro un gato negro que no está ahí, pues las excepciones no poseen ningún rasgo característico: son meras construcciones lingüísti$\mathrm{cas}^{30}$. Las excepciones son solo condiciones negativas del antecedente de una norma ${ }^{31}$. Si no es posible separar las excepciones del resto de condicionales, entonces las excepciones no tienen identidad propia ${ }^{32}$. De esta forma, una excepción es solo recurso retórico al momento de delimitar el ámbito de aplicación de una norma. Son, en este sentido, fragmentos del antecedente, sin características diferenciables.

La propuesta de Williams refleja las consecuencias de aplicar una representación lógica de las normas. Esta propuesta, para los fines del presente documento, es valiosa por dos razones: i) la noción de excepción puede ser empleada para clarificar un uso emotivo del lenguaje (tiene incidencia en una argumentación pragmática, a efectos de generar persuasión en el aplicador del derecho al momento de analizar la aplicabilidad de una norma); y ii) permite mostrar el rendimiento explicativo de la aproximación lógica sobre esta materia. Sin perjuicio de ello, hay aspectos relevantes que no están siendo esclarecidos como, por ejemplo, la diferencia entre los actos necesarios del demandado para que no sea aplicable la norma o la diferencia entre señalar «no hice» y «lo hice, pero...», anotada líneas atrás.

\section{ii. Excepción como reformulación normativa}

Frederick SCHAUer ha desarrollado una aproximación propia de qué es una excepción (en un sentido conceptual). Previo a profundizar el punto, hay que precisar qué entiende por norma. Este autor sostiene que las normas están compuestas por dos niveles: a) nivel prescriptivo o generalización de una justificación que posee una prescripción (norma condicional que contiene una orden o prohibición de realizar una acción); y b) nivel justificativo o de razones que sustentan las normas (justificación o propósito de las mismas, en adelante razones subyacentes ${ }^{33}$. En este sentido, la formulación

29. Williams, 1988: 278.

30. Williams, 1988: 278.

31. Cabe resaltar que Alchourrón ha desarrollado una propuesta muy relevante en relación a cómo dar cuenta de la reducción del alcance de las nromas desde una aporixmación lógica. Este autor analizó la idea de norma derrotable como una norma compuesta por un antecedente compuesto por condiciones explícitas y presupuestos implícitos. En este sentido, hemos de representar las normas incorporando un operador de revisión $(f)$ en el antecedente que de cuenta de dichos elementos, los cuales operan, conjuntamente, como una condición suficiente para el antecedente. Dada la complejidad de esta discusión profundizaré sobre ella en otro momento. Al respecto ver los textos contenidos en Alchourrón, 2010.

32. Desde esta aproximación, entonces, en la estructura de las normas no cabría diferenciar entre condiciones conjuntivas y condiciones de excepción (en clave de Susskind), pues solo tendríamos los primeros. Sobre este punto volveré líneas más adelante.

33. SCHAUER, 2004: 98.

Doxa. Cuadernos de Filosofía del Derecho, (2020), 43, pp. 427-456 
prescriptiva de la norma es una generalización de un propósito o justificación. De esta forma, la selección de propiedades (entendidas como identificadores de estados de cosas o de acciones) con las que construimos un antecedente de la norma está determinada por esta justificación (en otros términos, una norma sería una especificación de una justificación).

Al momento de analizar si una norma es aplicable a un caso individual puede suceder uno de los siguientes escenarios:

1) El caso individual está dentro de los usos lingüísticos de los términos empleados en el nivel prescriptivo y del ámbito de aplicación de las razones subyacentes.

2) El caso individual está dentro de los usos lingüísticos de los términos empleados en el nivel prescriptivo y fuera del ámbito de aplicación de las razones subyacentes.

3) El caso individual no está dentro de los usos lingüísticos de los términos empleados en el nivel prescriptivo, pero sí dentro del ámbito de aplicación de las razones subyacentes.

4) El caso individual está fuera de los usos lingüísticos de los términos empleados en el nivel prescriptivo y del ámbito de aplicación de las razones subyacentes.

El primer y cuarto caso son supuestos de aplicación y no aplicación respecto de los cuales no hay controversia. El segundo y tercer supuesto son casos en los que el antecedente contiene una inadecuada especificación del nivel justificativo. Schauer llama a estos supuestos "experiencias recalcitrantes» ${ }^{34} \mathrm{o}$ casos en los cuales tenemos razones fundadas en las razones subyacentes de la norma para variar el veredicto que se obtendría de aplicar el nivel prescriptivo. Estas se clasifican en supraincluyentes e infraincluyentes.

Las experiencias recalcitrantes supraincluyentes son aquellas en las cuales el caso individual es subsumible en el nivel prescriptivo, pero no es cubierto por el ámbito de aplicación del nivel justificativo. En este primer grupo de casos la controversia surge dado que el antecedente debería incluir más propiedades a efectos de que no regule el caso individual. Las experiencias recalcitrantes infraincluyentes, en segundo lugar, son aquellas en las cuales el caso individual no es subsumible en el nivel prescriptivo, pero sí es cubierto por el ámbito de aplicación del nivel justificativo. En estos casos el antecedente debe eliminar propiedades a efecto de ampliar el ámbito de aplicación en aras de regular todos los supuestos para los cuales fue formulada la dimensión prescriptiva.

Paso ahora a analizar si la noción de excepción esclarece estos puntos teóricos o no, en específico, las experiencias recalcitrantes supraincluyentes. Para ScHAUER existe una conexión entre la disponibilidad de herramientas lingüísticas por parte de los operadores y el uso del término «excepción». El nivel prescriptivo de las normas, esto es, su formulación lingüística, puede ser redactado de manera adecuada o inadecuada para captar el nivel justificativo. En aquellos casos que los operadores jurídicos consideren que la

34. Schauer, 2004: 98.

Doxa. Cuadernos de Filosofía del Derecho, (2020), 43, pp. 427-456 
formulación que es inadecuada, -es decir, carece de palabras adecuadas para delimitar el ámbito de aplicación de una norma ${ }^{35}$ - entonces harán uso de «excepciones» a efectos de que la regulación sea precisada respecto a los fines que se pretenden alcanzar. Bajo esta línea, la existencia de una excepción no es enteramente un producto de la casualidad lingüística, sino de la necesidad por expresar objetivos mediante palabras precisas (productos lingüísticos en términos de Schauer) ${ }^{36}$.

De acuerdo a esta propuesta, el uso de la noción excepción es meramente instrumental ${ }^{37}$, pues es dependiente de la interpretación que realice el operador de los términos contenidos en la formulación normativa. Si los usos lingüísticos y la estructura conceptual vigente de los términos empleados en el antecedente de la norma reflejan el mundo como ellos desean verlo (conforme a cómo entienden la dimensión justificativa) entonces no requerirán de usar «excepciones» para precisarlo. En cambio, cuando consideren que no es así, recurrirán al uso de excepciones.

La consecuencia de entender las excepciones de esta forma es que pierde sentido hacer una distinción entre una excepción de lo que se está exceptuando. No hay diferencia entre: i) señalar que la propiedad p contenida en el antecedente de una norma no es aplicable a una acción x; y ii) expresar que el antecedente de una norma está compuesto por la propiedad p y la exclusión de la acción x (representado como p. $\neg \mathrm{x}$ ). En el primer caso tenemos una norma N1que no regula un determinado supuesto; en el segundo caso tenemos una norma $\mathrm{N} 2$ cuyo antecedente es distinto, pero tiene el mismo ámbito de aplicación que N1. Ambas dicen lo mismo; solamente estamos eligiendo una forma distinta de explicarlo. Así, para Schauer, el concepto de excepción es vacío, pues no resultaría ser un concepto analíticamente diferente de la posibilidad de introducir en el sistema normativo una prescripción nueva por parte de los aplicadores del derecho (reemplazar N1 por N2, por ejemplo) ${ }^{38}$.

El autor analiza dos reglas para ejemplificar su tesis: i) la prohibición de aparcar vehículos en un parque (la cual refleja el objetivo de promover de la seguridad); y ii) la prohibición de ingresar con perros a un restaurante (la cual refleja el objetivo de prevenir disturbios en este tipo de espacios). Pensemos en circunstancias en que el intérprete considere que es necesario introducir una excepción: una ambulancia atendiendo una emergencia para la primera regla, y un perro lazarillo acompańando a un ciego para la segunda. En estos casos puede que le exijamos al aplicador del derecho que (asumiendo una interpretación literal de cada disposición), introduzca una excepción a las reglas no previstas en su literalidad. En este punto Schauer postula un criterio normativo para la introducción de excepciones: ¿̨cuándo debemos considerar que una excepción

\footnotetext{
35. He elegido esta redacción a efectos de guardar fidelidad con la exposición de SCHAUER, pero considero que el problema no está focalizado en la elección de palabras sino en la de códigos hermenéuticos mediante los cuales se elige o crea las normas.
}

36. SCHAUER, 1991: 878.

37. SCHAUER, 1991: 879.

38. SCHAUER, 1991: 893. 
es necesaria y cuándo no? Para SCHAUER debemos verificar que exista consistencia entre la prescripción normativa y la razón subyacente ${ }^{39}$.

De existir inconsistencia entre la prescripción normativa y la razón subyacente, entonces tendríamos una razón para modificar la que se consideraba que, hasta ese momento, era la prescripción normativa ${ }^{40}$. Hablar de excepciones solo sería un uso distractor de palabras, pues es solo otra forma para señalar que estamos reformulando la dimensión prescriptiva para adecuarla a la dimensión justificativa ${ }^{41}$ (pero en este supuesto, estaríamos aplicando la misma norma).

Cabe precisar que SCHAUER solo ha analizado el supuesto en el que una prescripción no sea coherente con su justificación. Dicho autor no ha analizado el supuesto en que una prescripción no sea coherente con otra norma del sistema normativo (o de otro sistema normativo aplicable y normativamente relevante para el caso). Dicho escenario fue analizado y teorizado por Claire Oakes Finkelstein quien, empleando el mismo marco conceptual, considera que la noción de excepción sí es útil para esclarecer las experiencias recalcitrantes.

Esta autora nos resume adecuadamente la postura de Schauer en dos hipótesis: a) hablar de excepciones es una preocupación estética y no conceptual; y b) las circunstancias que generan excepciones son especificaciones de la dimensión justificativa de la misma norma. En breve, de acuerdo a esta concepción, las prescripciones se han tragado las excepciones, pues todo es parte de un solo antecedente ${ }^{42}$. Más preciso aun: introducir una "excepción» sería equivalente a modificar la prescripción a la luz de sus razones subyacentes ${ }^{43}$.

Solo serían excepciones, desde este punto de vista, todas aquellas modificaciones a la prescripción que son realizadas considerando las razones subyacentes al momento de aplicar la regla a un caso concreto. ¿Es posible, entonces, diferenciar entre una modificación de la prescripción y la noción de excepción?

Pensemos en el caso en el que la razón subyacente de una norma N1 ha entrado en conflicto con la razón subyacente de una norma N2, y el conflicto es solucionado dándole prioridad a esta segunda razón subyacente (N2). Esto se traduce, normativamente, en que el antecedente de la norma N1 debe introducir una limitante que excluya la acción o hecho que será regulado por la norma N2. La noción de excepción daría cuenta de este tipo de casos: reducir el ámbito de aplicación de la prescripción como consecuencia de una ponderación entre razones subyacentes ${ }^{44}$.

39. SCHAUER, 1991: 894.

40. SCHAUER, 1991: 894.

41. SCHAUER, 1991: 895.

42. Finkelstein, 2000: 507.

43. Finkelstein, 2000: 511. Cabe precisar que esta aproximación de las excepciones solo se sigue de comprometernos con un modelo transparente o permeable de las normas y no con un modelo opaco. Schauer, 1998: 236-37.

44. Finkelstein, 2000: 511. Tendríamos dos momentos: i) identificar que para el caso individual la razón subyacente de la norma no da justificación para aplicar a consecuencia jurídica; y ii) a mérito de lo anterior,

Doxa. Cuadernos de Filosofía del Derecho, (2020), 43, pp. 427-456 
En este contexto, Finkelstein considera que Schauer nos pide hacer una distinción entre una situación limitante (que reduce el ámbito de aplicación de la consecuencia jurídica) prevista y agregar una nueva situación limitante en el antecedente. La primera es un elemento ya existente en la norma (es una especificación de la razón subyacente). La segunda, en cambio, si entendemos por nueva a una condición que no resulta de la especificación de la razón subyacente de la norma, sino de otra, entonces refiere a modificación de la norma ${ }^{45}$.

De este modo, para Schauer hablar de excepciones es lo mismo que hablar de cambiar las prescripciones, en consideración de un caso individual, en aras de generar mayor precisión respecto a cómo traducir las razones subyacentes en una estructura condicional y prescriptiva ${ }^{46}$-es decir delimita el uso del término «excepción» únicamente a un supuesto de deficiencia interna ${ }^{47}$ - . Ahora bien, ello dejaría fuera a los escenarios de deficiencia externa, es decir, aquellos casos en los que la regla es superada por exigencias externas tanto a la formulación normativa como a las razones subyacentes. Conforme a lo expuesto, el marco teórico de Schauer puede ser empleado para justificar la distinción entre una excepción y el resto de condiciones siempre que estemos en casos de deficiencia externa. Si bien expresamente el autor ha tratado de sustentar lo contrario, de sus propias tesis es posible formular reconstrucciones teóricas que permiten justificar la diferencia señalada. En ese sentido, es posible sostener que las tesis schauerianas no son propias de una aproximación retórica, sino conceptualista.

\section{b. Aproximación conceptualista}

De acuerdo con este conjunto de tesis, las excepciones no son reducibles a elementos negativos en el antecedente de la regla. Para quienes adoptan esta postura las excepciones poseen un contenido conceptual propio que les diferencia de otros condicionantes del antecedente. Al respecto, paso a reconstruir las propuestas de Claire OAKes Finkelstein, Luis Duarte d’Almeida y Bruno Celano al ser claros ejemplos de este conjunto.

Cabe volver a precisar que quienes sostienen una aproximación retórica no ven contenido conceptual en las excepciones, pues se compartan igual que el resto de condiciones: limitan el ámbito de aplicación de la norma. En cambio, quienes sostienen una aproximación conceptualista consideran que «excepción» posee contenido conceptual

reformular el ámbito de aplicación de la norma para que esta capture de manera más adecuada a la razón subyacente.

45. Finkelstein, 2000: 512.

46. Finkelstein, 2000: 514.

47. «Cuando una regla no es aplicable en aquellos casos en los que la justificación para esa regla es inaplicable, ella se vuelve inaplicable en virtud de una deficiencia interna (...) una regla es vulnerable a las deficiencias internas cuando resulta inaplicable sobre la base razones exclusivamente vinculadas con la aplicabilidad de las justificaciones que subyacen a la propia regla». SCHAuer, 2004, p. 179. 
clarificador de los antecedentes de las normas, pero no identificable mediante el análisis lógico-formal, sino en otros tipos de análisis de las normas.

\section{i. Excepciones como resultado de conflicto entre razones subyacentes}

Finkelstein, como ya he señalado líneas atrás, ha formulado una propuesta de conceptualización de excepción a partir de las ideas de Schauer. Esta autora se va enfocar, principalmente, en desarrollar la siguiente idea: las razones subyacentes pueden entrar en conflicto y ser superadas mutuamente -no generando el resultado del conflicto la invalidez de una razón subyacente- ${ }^{48}$.

Una de las posibles soluciones del conflicto entre razones subyacentes es crear excepciones: incluir nuevas propiedades en el antecedente de una de las prescripciones a efectos de reducir su ámbito de aplicación. En otros términos: se excluye de su regulación a cierto tipo de casos genéricos ${ }^{49}$.

Para esta autora la mejor manera de entender lo que es una excepción es a partir de la idea de deficiencia externa, es decir, considerando un conflicto entre una regla con otro elemento normativo diferente de las razones subyacentes de la regla ${ }^{50}$. Las excepciones, desde esta aproximación, son resultados de un conflicto entre razones subyacentes incompatibles. De esta manera, en caso de una deficiencia externa de la norma, el aplicador del derecho no aplica la norma, pero esta no deja de pertenecer al sistema normativo, sino que solo pierde aplicabilidad ${ }^{51}$. De este punto de vista, para la autora debemos realizar (usando el instrumental teórico de Schauer) la siguiente distinción conceptual ${ }^{52}$ :

48. Finkelstein, 2000: 514. Sobre este apartado debemos hacer dos precisiones: i) los principios operan como razones subyacentes a las reglas; y ii) entendemos que en caso de una regla entre en conflicto con un principio el operador resuelve una antinomia entre el principio que sustenta la regla y el principio con el que se ha generado el conflicto.

49. FinKeLSTEIN sostiene que una excepción debe ser creada en todos aquellos casos en los que la prescripción aplicable ya no puede determinar el resultado en concordancia con otra norma. Finkelstein, 2000: 514.

50. La autora precisa en este punto que, dependiendo de si se está de acuerdo con la tesis dworkiniana respecto a que los principios carecen de excepciones al no ofrecer aplicaciones concluyentes para la resolución de casos, entonces podremos hablar de conflictos entre reglas (postura que acepta la aludida tesis) o conflicto entre una regla y un principio (postura que no acepta la aludida tesis) Finkelstein, 2000: 515.

51. A este punto FinKelsTEIN considera que si asumimos que las reglas pueden entrar en este tipo de conflicto y que diferentes reglas pueden tener diferentes propósitos, entonces la conclusión de SCHAUER para considerar que toda excepción es un cambio de regla debido a una deficiencia interna no nos dice mucho. FINKELSTEIN, 2000: 516. Si las deficiencias de las normas fuesen solamente internas, no tendría sentido, desde esta aproximación, hablar de excepciones, pero no son el único escenario.

52. Finkelstein, 2000: 530-31.

Doxa. Cuadernos de Filosofía del Derecho, (2020), 43, pp. 427-456 
a) Condiciones limitantes como requisitos ${ }^{53}$ : da cuenta de las condiciones limitantes del ámbito de aplicación de la prescripción cuya incorporación se justifica en la razón subyacente de la misma norma.

b) Condiciones limitantes como excepciones: da cuenta de las condiciones limitantes del ámbito de aplicación de la prescripción cuya incorporación se justifica en razones subyacentes de otras normas.

Si Finkelstein está en lo correcto, entonces la representación de Williams de las excepciones como delimitadores de la norma (esto es, como condiciones negativas del antecedente) no es adecuada. Retomo los casos indicados líneas atrás: cómo diferenciar el no consentimiento en un caso de violación sexual de agresión no legal en un caso de lesiones. Desde esta aproximación conceptualista basada en la justificación de cada propiedad no podrían ser equiparables las condiciones negativas en un caso de violación con las de lesiones, desde el plano de justificación de la norma. En efecto, cada una de estas representaría la generación de razones subyacentes distintas (más preciso, del resultado de conflicto entre razones subyacentes).

Desde esta aproximación, las normas penales (por señalar un ejemplo) se construyen de manera que reflejen adecuadamente finalidades legislativas (regulación de la conducta, protección de bienes jurídicos, entre otros) $)^{54}$. Lo mismo con las excepciones, las cuales serían fragmentos de la norma que incorporan el cumplimiento simultáneo de otras finalidades legislativas al momento de aplicación de la norma. La distinción entre una excepción y el resto de condiciones, en este sentido, está en el plano de qué razón subyacente justificó su incorporación en el antecedente de la prescripción ${ }^{55}$.

\section{ii. Excepciones como resultado de la distribución de la carga de la prueba}

Luis Duarte D'Almeida ha ofrecido razones para considerar que es problemático no distinguir entre excepciones y condiciones negativas de la norma, pues no hacerlo induce a confusión ${ }^{56}$. Este autor, a partir de un estudio sobre cómo los procedimientos judiciales inciden en la aplicabilidad de las normas, realiza dos distinciones relevantes para entender la estructura de los antecedentes. En primer lugar, diferencia entre circunstancias positivas y negativas: «circunstancia positiva» se refiere a la descripción de la ocurrencia de ciertos eventos; en cambio «circunstancia negativa» se refiere a la descripción de la no ocurrencia de ciertos eventos.

53. En este punto he variado la nomenclatura empleada por FinKelstein a efectos de adecuarla a los términos que empleo en el presente documento.

54. Finkelstein, 2000: 531.

55. Como bien anotó un árbitro de este texto, la justificación de la introducción de una nueva propiedad (la excepción) sería un problema dogmático y no teórico. Sin embargo, la identificación de qué propiedades son excepciones si es un problema teórico.

56. DUARTE, 2015: 6. 
En segundo lugar, diferencia entre P-Facts y D-Facts: «P-Facts» se refiere a la conjunción de todas aquellas circunstancias (positivas o negativas) que, de ser probadas, hacen que una determinada decisión a favor del demandante se considere justificada; por su parte «D-Facts» se refiere a la disyunción de circunstancias (positivas o negativas) que, de ser probadas, son suficientes para prevenir que la decisión a favor del demandante esté justificada ${ }^{57}$. Así lo que necesita el demandante para que se decida, justificadamente, a favor suyo, es ofrecer la prueba de todos los P-Facts (en adelante P), sean estas circunstancias de tipo positivo o negativo. Pero si el demandando logra probar un solo D-Fact (en adelante D), entonces no se sigue la conclusión a favor del demandante.

Duarte, con este punto, pretende identificar condiciones de excepción. En este sentido, las excepciones son las $\mathrm{D}$, es decir, aquello que, de ser probado, hace injustificado que se decida a favor del demandante (dicho al revés, hace que esté justificado decidir a favor del demandado). El punto es representado por Duarte usando dos enunciados a demostrar: i) «si está probado $\mathrm{P}$ y no está probado ningún $\mathrm{D}$, entonces está justificado decidir a favor del demandante»; y ii) «si está probado P y está probado $\mathrm{D}$, entonces está justificado decidir a favor del demandado $»^{58}$.

Una circunstancia X puede ser probada o no probada, y lo mismo puede decirse de su negación ${ }^{59}$. En este sentido, nuestras posibilidades de prueba respecto de $\mathrm{X}$ son: $\mathrm{X}$ está probado; $\neg \mathrm{X}$ está probado; X no está probado; $\mathrm{o} \neg \mathrm{X}$ no está probado ${ }^{60}$. De estas posibilidades es relevante resaltar la diferencia entre «lo no probado» de «haber probado que algo no ocurrión: como una negación externa (no se ha probado D) y como una negación interna (se ha probado $\neg \mathrm{D}$ ), respectivamente.

Para Duarte, la noción de excepción se explica adecuadamente empleando «no está probado ningún D», es decir, entendiéndola como una negación externa (no se ha probado D). Como bien ejemplifica el autor, un aplicador del derecho no puede conceder una compensación por incumplimiento contractual en caso que algún $\mathrm{D}$ sea probado. La prueba de $\mathrm{D}$ hace inviable decidir a favor del demandante de manera justificada ${ }^{61}$. En efecto, para que se decida justificadamente a favor del demandante, además de haber probado $\mathrm{P}$, tiene que ser el caso que ninguna $\mathrm{D}$ haya sido probada ${ }^{62}$.

57. Duarte, 2015: 17. Dicho de otro modo, que la decisión a favor del demandado esté justificada.

58. Duarte, 2015: 52.

59. En este punto Duarte no aclara qué quiere decir con probar que un hecho no ocurrió. Sin perjuicio de ello, asumiré, a efectos de no desviar la atención de los puntos de interés sobre su texto, que esta es una forma de expresar la prueba de hechos incompatibles con X, de manera que no sería posible que $\mathrm{X}$ haya ocurrido.

60. DuARTE, 2015: 53.

61. Duarte, 2015: 54 .

62. En este punto DuARTe precisa, adecuadamente, que predicar que no está probado P a efectos de que esté justificado decidir a favor del demandante (asumiendo que ha probado P) tiene dos implicaciones considerando la negación externa e interna de D:

1. Decir que si $\mathrm{D}$ está probado entonces el juez no puede decidir a favor del demandante, es decir, no puede ser el caso que simultáneamente se haya probado P y D y esté justificado decidir a favor del demandante. Este fraseo captura la negación externa de D: el juez deberá decidir a favor del demandante si es el caso que este probó $\mathrm{P}$ y no está probada ninguna $\mathrm{D}$. 
En relación a esta última idea, el demandante requiere de la conjunción de tanto $\mathrm{P}$ como de la no prueba de $\mathrm{D}$. En otros términos, «Si P está probado y no está probado $\mathrm{D}$, entonces está justificado decidir a favor del demandante» ${ }^{63}$.

Pero también puede ser el caso que el demandante haya podido probar $\neg$ D. Esta última posibilidad es esclarecida mediante la negación interna de D. Estos casos son entendidos como parte del conjunto de circunstancias (positivas o negativas) que, de ser probadas, generan que esté justificado decidir a favor del demandante (pero esta sería una forma encubierta de llamar a un elemento de P).

A partir de estos puntos, Duarte formula su propuesta: los D identifican los hechos que operan como excepciones. Para que un hecho sea $\mathrm{D}$, debe ser el caso que: i) de ser probado el hecho $\mathrm{X}$ (sea este una circunstancia negativa o positiva), entonces estará justificado decidir a favor del demandado; y ii) la prueba de $\neg X$ no sea necesaria para que la decisión esté justificada a favor del demandante. Dicho de otro modo: los P son hechos cuya prueba es necesaria para que una decisión esté justificada para el demandante, mientras que los $\mathrm{D}$ son hechos: i) cuya prueba impide que la decisión a favor del demandante esté justificada; y ii) que no son incompatibles de $\mathrm{P}^{64}$.

Desde esta aproximación, como podemos ver, la identificación de las excepciones está vinculada con la forma en que está distribuida la carga de la prueba en procesos de toma de decisiones jurídicas. No es un problema de contenido conceptual de los términos empleados, del tipo de inferencia ni de las condiciones del antecedente, sino de cómo están diseñadas las reglas que generan cargas de la prueba.

De este modo, la identificación de las D (esto es, de las excepciones) se hace a partir de todos aquellos que hechos que, de ser probados por el demandado, entonces hacen que esté justificado que se decida a favor de este ${ }^{65}$. En relación a lo visto hasta el momento, paso a resumir, conforme a la propuesta de Duarte, en qué momento está justificado decidir a favor de cada parte ${ }^{66}$ :

1) Decisión justificada a favor del demandante: que la proposición sobre hechos P esté probado y ninguna proposición sobre hechos D esté probada.

2. Decir que el demandante tendrá éxito si este puede probar que no ocurrió una D. Este fraseo captura la negación interna de D: el juez deberá decidir a favor del demandante si es el caso que este probó P y probó que no ocurrió $\mathrm{D}$.

DUARTE, 2015: 54 .

63. Duarte, 2015: 55.

64. Duarte, 2015: 77. El punto ii) insiste en que probar D no es lo mismo que probar $\neg$ P. Como ya hemos líneas atrás, una excepción da cuenta de un hecho correlativo a la ocurrencia de los P, pero que excluye las consecuencias jurídicas vinculadas a estos.

65. Cabe indicar que DuARTE comprende la noción de carga de la prueba como requerimientos a asegurar que ciertas proposiciones están probadas. Sobre este punto no profundizaré, pues no es necesario para dar cuenta de la conexión entre identificación de excepciones con D. Duarte, 2015: 92.

66. Duarte, 2015: 92. Duarte, en este sentido, tendría una comprensión material de la carga de la prueba, pues el pretende dar cuenta del tipo de clasificación que se debe dar ante determinados hechos probados y/o no probados. 
2) Decisión justificada a favor del demandado: que la proposición sobre hechos $\mathrm{D}$ esté probada o que ninguna proposición sobre hechos $\mathrm{P}$ esté probada.

En vista de dicho punto, la carga de la prueba depende de lo que necesite cada parte para tener éxito procesal (esto es, que esté justificado que se decida a favor suyo), lo cual puede incluir que ciertas proposiciones estén probadas, como que ciertas proposiciones no estén probadas ${ }^{67}$.

La aproximación probatoria de las excepciones, como hemos visto, ofrece una propuesta conceptualista que justifica la distinción entre requisitos y excepciones. Hay dos ideas de trasfondo que sustentan esta tesis. La primera, es que solo tiene sentido hablar de excepciones en contextos adversariales. Predicar que una norma contiene excepciones es resultado de una forma de entender la aplicación de normas en las que hay dos partes que discuten entre sí para que una norma se aplique/no se aplique. No tiene sentido hablar de excepciones en contextos no adversariales. La segunda, es que la identificación de excepciones a las reglas procedimentales sobre prueba (dicho con más precisión, a las reglas de la carga de la prueba entendida como reglas de juicio ${ }^{68}$ ). Si cambia la distribución de la carga de la prueba cambian, también, que propiedades del antecedente operan como condiciones de excepción.

\section{iii. Excepciones como inaplicación de resultados absurdos}

Bruno Celano ha formulado una tesis para diferenciar entre lo que él considera usos adecuados del término «excepción», de usos no adecuados. Un uso adecuado del término «excepción» es aquel empleado para dar cuenta de la no aplicación de la norma en vista a que tenemos razones para considerar que no es razonable aplicar al caso individual el consecuente normativo.

A efectos de analizar esta propuesta es necesario partir por las ideas esgrimidas por este autor respecto de las tesis justificativas vistas anteriormente. Retomando el punto, quienes analizan las formulaciones normativas como generalizaciones de las razones subyacentes (dicho de otro modo, quienes consideran que las normas están compuestas por dos niveles) consideran que en caso de resolver una experiencia recalcitrante mediante la incorporación de nuevas propiedades en el antecedente de la formulación normativa están sosteniendo que el resultado no es cambiar de norma, sino que es la misma norma solo que mejor comprendida. A esta idea la denomina la tesis de la suposición de identidad. De acuerdo con Celano, quienes adoptan esta tesis sostienen que introducir limitaciones en el antecedente a afectos de resolver una experiencia

67. Duarte, 2015: 91-92. Debo precisar que, conforme a la postura de Duarte, el demandado no tiene el deber de probar ninguna proposición. Al demandado no le corresponde probar $\neg \mathrm{P}$ (si lo hace es por razones tácticas) y solo tendrá la carga de probar $\mathrm{D}$ en caso el demandante haya podido probar $\mathrm{P}$. En este contexto que el demandado tenga como condición de éxito que ciertas proposiciones no estén probadas refiere a que este pueda o bien se tenga por probado $\neg \mathrm{P}$ o bien consiga probar una $\mathrm{D}$.

68. FERNÁNDEZ, 2004: 134-36. 
recalcitrante, no desvirtúa la identidad a pesar de que el antecedente haya variado. Esta suposición de identidad tiene dos posibles versiones. La primera versión vincula el uso de excepciones con la estrategia especificacionista. Conforme a este método, ante dichos escenarios lo que debemos hacer es entender la norma de manera más profunda, es decir, ser más finos respecto a cómo está compuesto el antecedente de la norma a luz de su dimensión justificativa ${ }^{69}$. La segunda está conectada con la noción de normalidad. La forma en que se reduce el ámbito de aplicación en cada una de estas versiones nos permite dar cuenta de usos adecuados y de usos inadecuados de la noción de excepción. Analizo cada una por separado.

En primer lugar, podemos expresar una norma de diferentes maneras, es decir, mediante diferentes formulaciones. Algunas pueden capturar más detalles, otras menos. Siguiendo el método especificacionista ${ }^{70}$, esto es, introducir precisiones en el antecedente, no estamos modificando la norma, sino su formulación inicial (en el nivel prescriptivo). Es decir, no variamos de norma, sino su comprensión: pasamos de un entendimiento parcial e incipiente de la norma a uno más fino y adecuado con lo que es la norma ${ }^{71}$.

De esta discusión Celano resalta que, desde dicha aproximación a la suposición de identidad, la solución a casos recalcitrantes generaría excepciones prima facie de la norma. Un estudio de la norma más preciso llevaría a que estos casos estarían previstos por la norma de manera implícita: la norma puede refinarse para permitir un caso recalcitrante como excepción (Si p entonces q, excepto cuando p.z $)^{72}$.

La segunda versión de la suposición de identidad considera que dicha tesis está conectada con una noción de normalidad, de lo cual se sigue podamos diferenciar entre supuestos normales ${ }^{73}$ y anormales de aplicación de la norma. En este punto Celano opta por entender «normal» como expectativas sobre qué es o cómo debiera ser un

69. Celano, 2012: 271.

70. Como bien lo resume Celano, el especificacionismo nos propone lo siguiente: al enfrentar un conflicto o en la aplicación de una norma relevante a un caso que lleva a una solución considerada inapropiada o insatisfactorias (por un juicio de valor) debemos especificar, esto es, restringir el ámbito de aplicación, de una de las normas en conflicto. Entiéndase por «especificar» la inclusión de nuevas condiciones en el antecedente (clausulas que establezcan el dónde, cuándo, porqué, cómo, bajo qué medios, a quién, el por quiénes se debe o no debe ejecutar una acción u otras) a efectos de hacer desvanecer el conflicto. Celano, 2018: 270.

71. Celano, 2012: 272. Cabe indicar que esta idea está vinculada con la pretensión del especificacionismo de listar todas las condiciones limitantes, pero eso es problemático dado que es un objetivo inalcanzable. Las razones en: Celano, 2012, pp. 275-76 y en Celano, 2009, p. 239. Asimismo, Miller, 1956: 267, Bouvier, 2012: 258-262, у НАRT, 2009: 160, por señalar algunos. No profundizaré en este punto dado que escapa los fines del trabajo.

72. Celano, 2012: 277.

73. Cabe precisar que "supuesto de normalidad» es altamente ambiguo. Entre los muchos posibles sentidos que le podemos atribuir a estos términos he identificado los siguientes (lista no es exhaustiva, sino únicamente ejemplificadora del problema de ambigüedad):

1. Entendida como expectativa injustificada, es decir, como casos en los cuales se asume que un hecho ocurrirá, a pesar de que no tenemos garantías de ello pase (por ejemplo, vemos huevos de peces y pensamos que habrá peces, sin conocer la tasa estadística de éxito de cuantos peces es probable que nazcan en las circunstancias en las que se encuentran esos huevos). 
hecho o un comportamiento. Es en este contexto (es decir, bajo la presuposición de determinadas condiciones de normalidad), podemos afirmar que las normas controlan nuestra conducta vinculando soluciones normativas con tipos de casos posibles ${ }^{74}$.

A partir de la reconstrucción de las versiones de la tesis de suposición de identidad, Celano identifica dos usos del término «excepción». En primer lugar, desde el especificacionismo se realiza un uso prima facie o falso de excepciones. Ello agrupa a todas aquellas aproximaciones que consideran que es posible considerar que las limitaciones al ámbito de aplicación de la norma ya están incorporadas de manera implícita (sean especificables de su dimensión justificativa). En este sentido, quienes proponen que una excepción es el resultado de refinar la prescripción de manera que los principios subyacentes sean mejor concretizados están predicando falsas excepciones. En efecto, una comprensión o formulación adecuada de la norma podría, y de hecho lo haría, ponerlas en pie de igualdad con otras condiciones ${ }^{75}$.

En este punto creo que es oportuno anotar que el uso falso de excepción como resultado de una especificación, como lo reconstruye Celano, es una conclusión no predicable a todos los supuestos. El autor está dando cuenta de las reformulaciones

2. Entendida como habitualidad, es decir, como una generalización a partir de tener información sobre cierta frecuencia de que un determinado hecho ocurre luego de ciertas circunstancias.

3. Entendida como afirmación no cuestionada. Esto en un sentido prescriptivo y descriptivo. Prescriptivo por cuanto sea una afirmación que, dentro del espacio público, no se admite poner en duda (por ejemplo, está mal devorar niños recién nacidos). Descriptivo por cuanto sea una afirmación que no haya sido, hasta el momento, polemizada en espacios públicos.

4. Entendida como un modelo ideal. En otros términos, como referencia a una propuesta de deber ser.

5. Entendida como un modelo regulativo. Refiere al tipo de supuesto habitual que es empleado para diseńar marcos normativos a todos y todas (por ejemplo, este sentido es empleado por la literatura crítica que sostiene que el derecho de un país ha sido pensado a partir de los problemas de una persona que es hombre, entre 20 y 50 años, heterosexual, sin ningún tipo de discapacidad, caucásico, católico, económicamente autónomo, entre otros).

6. Entendida como estándar de conocimiento. Refiere a aquellos criterios que son empleados para evaluar el tipo de información que una persona debiera poseer, en función a las circunstancias fácticas en la que habitualmente se encuentra (por ejemplo, el estándar de consumidor razonable empleada en protección al consumidor).

7. Entendida como estándar de evaluación. Refiere a todos ellos criterios que son empleados como criterios de corrección de moral social.

74. Celano, 2012: 287.

75. Celano, 2012: 281. Las excepciones prima facie también alude a las excepciones que se entienden como el resultado de conflicto entre normas al momento de crear una preferencia (o jerarquía) entre las normas (por presuponerse una regla de prioridad). La dicotomía explícita-implícita, para esta tesis, no es clarificadora. Para responder a la pregunta de si las excepciones implícitas son o no verdaderas excepciones, siguiendo a CeLANO Y A BRIGAGLIA, tenemos dos posibilidades: i) lo son siempre que por estas entendamos el uso de cláusulas vacías («a menos que existan razones concluyentes en contra» por ejemplo), que mantengan reflectores sobre la posibilidad de anormalidad; ii) si se emplea para referir a una propiedad de la norma, en una versión mejor entendida de esta, entonces: o bien la regla no ha sido aplicable en su primera versión (de esto no se sigue una excepción); o bien estamos creando una nueva regla, diferente de la original, que es más específica. Celano y BRIGAGLIA, 2018: 134-35. Para ilustrar el último punto ofrece el siguiente ejemplo: Tenemos una norma «todos los ciudadanos mayores de edad tienen derecho a votar» y una excepción implícita que excluye de dicho derecho a los mayores de edad que sean interdictos. Ante tal escenario formulamos una nueva norma: «todos los ciudadanos mayores de edad no interdictos tienen derecho a votar». Celano, 2017: 100.

Doxa. Cuadernos de Filosofía del Derecho, (2020), 43, pp. 427-456 
de la dimensión prescriptiva de la norma como producto de haber solucionado una generalización no satisfactoria de la razón subyacente. Esto puede ser entendido dos formas: como deficiencia interna de la norma o como deficiencia interna de la norma en relación a un sistema normativo.

Como deficiencia interna de la norma refiero a los casos de solución de experiencias recalcitrantes en los que la formulación normativa varía a efectos de reflejar de mejor manera la razón subyacente. En este punto las ideas de Celano, considero, son bastante acertadas.

Como deficiencia interna de la norma en relación al sistema refiero a dos casos. Por una parte, se puede producir un conflicto de razones subyacentes de diferentes normas que pertenecen al mismo sistema normativo, lo que conduce a especificar (más) la formulación normativa de una de las normas a efectos de representar la ordenación entre estas (la solución al conflicto). En este caso, la conclusión de Celano (uso prima facie de excepciones) puede ser entendida a manera de que estamos entendiendo, de mejor manera, el sistema normativo, por lo que esta misma conclusión se sigue.

Por otra parte, se puede producir un conflicto de razones subyacentes de diferentes normas que pertenecen a sistemas normativos diferentes, lo que conduce a especificar (más) la formulación normativa de una de las normas a efectos de representar la ordenación entre estas (la solución al conflicto). En estos supuestos, la conclusión de Celano no podría seguirse, pues no estamos en el caso de que entendemos mejor la norma (refleje de mejor manera sus razones subyacentes). La especificación no se sigue de un mejor entendimiento ${ }^{76}$, sino de haber ordenado prescripciones que forman parte de sistemas diferentes en el que un sistema introduce valoraciones y preferencias no consideradas en otro sistema. En este sentido, la especificación no tiene como resultado, únicamente, falsas excepciones.

En segundo lugar, en relación a la tesis de suposición de identidad conectada con una noción de normalidad, predica un uso «verdadero» de excepciones. Para identificar las excepciones verdaderas ${ }^{77}$ debemos abordar la distinción entre casos normales y casos anormales. Los casos normales son todos aquellos supuestos en los cuales es razonable seguir la norma. Los casos anormales, en cambio, se refieren a que es razonable preguntarse si la consecuencia normativa prevista por la norma es, dadas las circunstancias, el curso de acción que debiera seguirse o que es correcto (desde algún criterio de corrección axiológico $)^{78}$. En este sentido, un caso anormal es una situación en la que se debe

76. Las tesis de CELANo en este punto podrían expresarse señalando que el autor considera que la introducción de falsas excepciones es producto de mejores descripciones. Pero en este caso no estamos ante una mejor descripción de la norma, sino ante una crítica a la norma formulada desde otro sistema normativo (el que nos sirve para realizar juicios valorativos de corrección, por ejemplo, un sistema axiológico).

77. Teóricamente esta propuesta sobre excepciones verdaderas se sitúa en un punto medio de dos extremos: i) por un lado las excepciones previstas en el antecedente de la norma; y ii) por el otro, que un juez no aplique una norma como resultado de una decisión discrecional. Celano, 2012: 282.

78. Celano, 2017: 98. Las reglas, de esta manera, se aplican (directamente dictan el veredicto correcto a ser adoptado por el juzgador pues es lo que la regla prescribe) solo en casos normales. en casos anormales el juzgador, si es razonable, no sigue la regla, la reconsidera. Celano y Brigaglia, 2018: 135. 
(tesis normativa) dar una excepción verdadera. Estas se dan en vista de dos condiciones: i) el caso recae bajo el antecedente de la norma; y ii) no es razonable, en dicho caso, adoptar la solución normativa prevista por la norma ${ }^{79}$. La calificación de no razonable conlleva a que el decisor (que se asume una persona razonable e informada) concluya que debemos actuar diferente a lo prescrito por la norma.

Reformulando: la noción de excepción verdadera refiere a la situación de tener un caso que posee la propiedad C, la cual está prevista en el antecedente de una norma, pero la consecuencia normativa no se sigue porque esta solo se aplica en circunstancias normales o ceteris paribus y las circunstancias en las que se ha dado $C$ no son normales. A mérito de esta propiedad es razonable que el juzgador se pregunte si el veredicto prescrito por la regla es el correcto y que tenga como respuesta que debe resolver el caso de manera incompatible a lo prescrito por la norma ${ }^{80}$.

El criterio de razonabilidad para diferenciar efectos razonables y no razonables de una norma, para Celano, es psicológico ${ }^{81}$. Todos poseemos un mecanismo inconsciente, denominado «el guardián», cuyas funciones son: i) verificar si el veredicto prescrito por las reglas coincide con algún parámetro inconsciente (intuiciones y expectativas de lo que entendemos por normal); y ii) si no es el caso, inhibir la aplicación de la regla, lo que activa la reconsideración (implementación de una excepción verdadera).

Los escenarios inconscientes, que funcionan como condiciones de normalidad, dan contenido a lo que sería una causal para una excepción verdadera. Desde esta aproximación, un signo confiable de una excepción verdadera es la sorpresa y la duda por parte del que toma las decisiones. Si no hay sorpresa y duda, entonces, no hay caso anormal, por tanto, no hay verdadera excepción ${ }^{82}$.

Un punto no esclarecido por Celano, pero relevante, es quién justifica la no razonabilidad de una consecuencia normativa. Una posibilidad es que sea el propio aplicador del derecho quien, con independencia de lo que aleguen las partes procesales, decida por sus propias convicciones considerar inaplicable la norma. Otra posibilidad es que recaiga en el demandado la responsabilidad de ofrecer al aplicador del derecho de las razones por las cuales no es razonable que se siga la consecuencia normativa. Esta última posibilidad resulta de interés, pues permite poner de relieve un rasgo compartido entre todas las concepciones de la aproximación conceptualista: aclaran cuáles son las posibilidades de actuación del demandado en un proceso judicial.

79. Celano, 2017: 99.

80. Celano y Brigaglia, 2018: 138.

81. Esto requiere de precisión. Celano y BRigaglia distinguen entre estados mentales y procesos mentales que se acceden de manera consiente o inconsciente (no accesibles mediante un estado consiente). Los conscientes son los que experimentamos y somos capaces de reportar dicha experiencia. Los inconscientes aluden a los estados y procesos mentales que no podemos experimentar directamente, pero que pueden ser descritos. Los estados mentales y los procesos mentales se desarrollan gracias a mecanismos inconscientes. ej.: las intuiciones son resultados conscientes de procesos inconscientes. Celano y Brigaglia, 2018: 136-37.

82. Celano y Brigaglia, 2018: 139. 


\section{EXCEPCIÓN Y EXCEPTUAR}

Las diferentes posturas teóricas analizadas anteriormente nos permiten mostrar que el análisis conceptual sobre qué son las excepciones permite aclarar diversas discusiones teóricas. Qué se esclarece mediante las diversas propuestas de conceptualización de «excepción» se ordena adecuadamente mediante la distinción entre, por un lado, predicar que una condición es una "excepción" y, por el otro, calificar a un conjunto de actos como «exceptuar» ${ }^{83}$ una norma. Al hablar de «excepción» se está dando cuenta de un tipo de condición, a efectos de predicar que esta posee rasgos que la diferencian del resto de condiciones en el antecedente (posee un efecto excluyente, como bien precisé al inicio del documento). En cambio, al hablar de «exceptuar», se hace referencia a las diversas operaciones jurídicas realizadas por el aplicador del derecho para incorporar una nueva condición limitante en el antecedente. Veamos este punto con más detalle.

\section{1. «Excepción»}

Predicar que en una norma hay una «excepción» refiere a las razones para diferenciar la operatividad de una determinada propiedad contenida en el antecedente. Las razones son formuladas considerando la justificación de la incorporación de la propiedad (SChauer-Finkelstein) o los efectos de la carga de la prueba (DuARte).

Si seguimos un análisis de la estructura de las normas desde la tesis de la justificación, entonces son excepciones las condiciones producidas de haber resuelto un conflicto entre razones subyacentes de dos normas diferentes (a efectos de evitar la crítica de Celano: conflicto entre una norma que pertenece a un sistema jurídico con una norma que pertenece a un sistema extrajurídico).

Analizada como una tesis sobre la identificación de condiciones dentro de un antecedente esta propuesta es problemática. Lleva implícita la idea de que entre una condición de aplicación conjuntiva y una condición de excepción se comparten todos los rasgos, salvo la explicación de su incorporación, pero el contenido de esa explicación depende de cómo el estudioso o el aplicador del derecho reconstruya la norma. De esta forma, la rentabilidad explicativa de esta propuesta radica en describir las operaciones que realizamos para crear la excepción antes que en la clarificación de qué es y cómo opera una condición de excepción.

En cambio, si seguimos la tesis de la distribución de la carga de la prueba, se esclarece qué condiciones son excepciones (en contextos adversariales), al asignarles un

83. Esta misma idea podría, en parte, ser explicada señalando que «excepción» padece de la ambigüedad actividadproducto. «Excepción» como actividad da cuenta de las actuaciones y razones ofrecidas para incorporar una condición limitante en el antecedente de la norma. En cambio, «excepción» como producto da cuenta de la condición limitante prevista en el antecedente de una norma. Sin embargo, no empleo dicha distinción, pues una excepción-producto no necesariamente es el resultado de una excepción-actividad (de haber exceptuado), como precisaré en este apartado, de manera que prefiero utilizar otra estrategia explicativa a efectos de no inducir a confusión. 
efecto excluidor de ser verificadas. Al respecto cabe resaltar lo siguiente: i) al identificar cómo está regulada la carga de la prueba podemos diferenciar entre qué propiedades son requisitos (las que deben ser probadas por el demandante) y cuáles excepciones (las que deben ser probadas por el demandado); ii) la excepciones son producto de cómo operan las metanormas de aplicación (entendiendo a la carga de la prueba como como reglas de calificación de veracidad de enunciados descriptivos de hechos); y iii) al variar la carga de la prueba de una determinada norma varían, a la vez, qué condiciones son las excepciones.

\section{2. «Exceptuar»}

Predicar que en una serie de actuaciones califican como «exceptuar» una norma, permite esclarecer diversas formas de resolver conflictos entre normas y conflictos entre niveles de legalidad. Por «conflicto entre normas» refiero a los casos en los cuales dos normas -pertenecientes a un mismo sistema normativo o dos sistemas normativos diferentes (uno jurídico y el otro extrajurídico), pero ambos aplicables y normativamente relevantes a un caso- ofrecen, bien estados de cosas incompatibles entre sí, bien guías de conducta irrealizables a la vez por el mismo sujeto.

En cambio, por «conflictos entre niveles de legalidad» doy cuenta de la incompatibilidad entre la prescripción y la justificación de una misma norma. Refiero a los supuestos en los cuales se asume que una prescripción normativa debe adecuarse a sus razones subyacentes.

Tanto en el caso de conflictos entre normas como en el de niveles de legalidad, el punto a aclarar es el mismo: una de las formas de solucionar un conflicto es reduciendo el ámbito de aplicación de una norma mediante la incorporación de una nueva condición, esto es, haciendo el antecedente más fino.

Esto muestra que la pregunta sobre cómo exceptuamos se responde en función a la teoría del derecho que estemos adoptando. En este sentido, considero que es pertinente conceptualizar la noción de «exceptuar» como la modificación de la aplicabilidad interna de la norma, esto es, como la operación de introducir nuevas propiedades que reducen el alcance del caso genérico previsto en el antecedente. Esta definición nos permite, desde un punto de vista metateórico, dar cuenta de las diversas propuestas de reconstrucción de qué hacen los operadores jurídicos cuando exceptúan las normas jurídicas.

La noción de «exceptuar» como actividad para solucionar un conflicto da cuenta de los supuestos en los cuales una antinomia es disuelta mediante la incorporación de una nueva condición limitante en el antecedente. Sigo el ejemplo propuesto por RodríGUEZ: imaginemos un sistema normativo en el que una norma N1 prescribe que, si alguien comete un homicidio, entonces es obligatorio sancionarlo $(\mathrm{p} \rightarrow \mathrm{Oq})$. Pero además de esta tenemos otra norma N2 que ordena que si alguien es menor de edad entonces es obligatorio no sancionarlo $(\mathrm{r} \rightarrow \mathrm{O} \neg \mathrm{q})$. En caso que tengamos una menor 
de edad que comete homicidio (p.r) tendríamos una contradicción. En este supuesto los operadores jurídicos, para poder resolver dicho caso, debieran introducir una regla de preferencia entre ambas a efectos de que, por ejemplo, prevalezca la solución de N2. En este supuesto, la norma aplicable al caso sería «quien cometa homicidio y no sea menor de edad, entonces debe ser sancionado» $(\mathrm{p}.\urcorner \mathrm{r} \rightarrow \mathrm{Oq})^{84}$.

Cabe precisar que esta reconstrucción nos aclara que quienes sostienen que hay normas que exceptúan a otras (se identifican normas de excepción) solo están dando cuenta de un conflicto normativo y de su solución, pero no proponen criterios de identificación de normas. Esta sería, bajo esta propuesta, solo una forma de explicar cómo se ha creado la excepción.

Por otra parte, exceptuar como solución a un conflicto de niveles de legalidad, clarifica un tipo de resultado en caso de solucionar un supuesto en el cual la consecuencia de una norma nos parece no razonable para el caso individual o supuestos de experiencias recalcitrantes ${ }^{85}$. Para dar cuenta del primer supuesto paso a reformular la propuesta de Celano: disponemos de un sistema normativo extra jurídico que nos ofrece un criterio de corrección que diferencia entre resultados razonables/no razonables. Las normas de dicho sistema tienen una jerarquía material superior a las normas jurídicas. En caso las consecuencias jurídicas sean no razonables, entonces se debe adecuar la norma jurídica a efectos de que sea razonable. Ello se logra introduciendo condiciones limitantes dentro del supuesto de hecho de la norma jurídica, es decir, exceptuando la norma jurídica.

En relación a las experiencias recalcitrantes supraincluyentes, como ya he dado cuenta, exceptuamos una norma al momento de resolver un conflicto entre razones subyacentes (sean estas pertenecientes al mismo sistema normativo o a diferentes sistemas normativos). El resultado de esta operación es la incorporación de una condición limitante en la dimensión prescriptiva de la norma con menos peso.

Estos supuestos nos permiten poner de relieve que «exceptuar», además, permite esclarecer que la aplicabilidad de una norma opera a manera de razón para la acción (para aplicar la norma a un caso) que puede entrar en conflicto con razones para la

84. «[P]ara que pueda hablarse de que una regla hace excepción a otra se requiere: a. Que las soluciones normativas de ambas resulten lógicamente incompatibles para cierto caso genérico; b. Que se estipule una preferencia de la solución normativa de una de ellas sobre la otra para evitar la contradicción». RoDRíGuEz, 2003: 86. En este mismo sentido Mendoca, 2012: 204. Una manera diferente de presentar este punto es mediante los estudios de la lex specialis. Siguiendo a Silvia Zorzetto, el carácter especial y general de una norma son rasgos lógico-conceptuales relacionales de los conceptos contenidos tanto en el antecedente como en el consecuente de la norma. En este sentido, una norma es especial respecto de otra a mérito de una relación de implicación lógica de los conceptos empleados por cada una de estas. En casos de antinomia la lex specialis puede ser entendida como un criterio interpretativo de manera a que siempre que sea identificada una relación entre dos interpretaciones inconsistentes entre sí (una con carácter general y otra con carácter especial entre sí), ello supone que debemos realizar una reinterpretación de las disposiciones normativas cuyo producto sea un microsistema de normas consistente. Al respecto ver ZorZetTo, 2010: 84-85, ZoRZetTo, 2013: 87.

85. Debo precisar que esta no es la única opción teórica disponible (podríamos incluir, por ejemplo, la noción de laguna axiológica), pero resalto solo esa a efectos de vincular «exceptuar» con las tesis de FinKelsTeIn y Celano explicadas en el presente documento. 
acción contrarias y ser superad ${ }^{86}$. Como podemos ver, la aplicabilidad de una norma, en situaciones de conflicto, no es una razón absoluta, sino solo una razón contribuyente.

La aplicabilidad de una norma, como razón contribuyente, puede darse en tres escenarios:

i) Una cierta norma es aplicable y ello se da sin que concurra ninguna otra razón que se oponga;

ii) Una cierta norma es aplicable, pero concurre con razones en contra de la aplicación y estas son razones no concluyentes; o

iii) Una cierta norma es aplicable, pero concurre con razones en contra de la aplicación y estas son razones concluyentes.

El primer escenario de aplicación de una norma no es problemático. Da cuenta de los casos de aplicación de una norma sin que se presente una situación conflictiva ni duda de su aplicabilidad.

El segundo escenario y tercer escenario dan cuenta de la misma situación, pero desde perspectivas diferentes. Ambos refieren a un caso de conflicto normativo en el que cada norma es aplicable (ofrece razones contribuyentes para su aplicación). El resultado es que una de estas normas en conflicto se aplica y la otra no se aplica (una de estas ofrecerá una razón concluyente). El segundo escenario es el de la norma que se aplicará al caso y el tercer escenario da cuenta de la norma que no se aplica y que, por tanto, se exceptuará ${ }^{87}$.

Las excepciones, en estos escenarios, clarifican los casos en los cuales consideramos que las razones para no aplicar la norma son concluyentes (este es el caso, por ejemplo, de lo que pretenden aclarar las propuestas de Celano y Finkelsetin).

Conforme a este punto podemos ver que la rentabilidad explicativa de «excepción» y de «exceptuar» radica en el punto teórico que pretendemos aclarar, pero no conviene, desde la reconstrucción propuesta en este documento, confundirlos. En efecto, la actividad de exceptuar no necesariamente arroja como resultado lo que nuestro criterio de identificación diría que es una condición de excepción. Por ejemplo, si exceptuamos como resultado de adecuar una prescripción a nuestro criterio de razonabilidad, ello no necesariamente se corresponderá con la carga probatoria del demandado para dicho caso.

Tras dar cuenta de algunas propuestas teóricas sobre la noción de excepción, ya es posible afirmar que con esta no se pretende resolver la pregunta de qué es una norma, sino de un tipo de condición prevista en el antecedente de una norma; es, por tanto, un problema de estructura de normas. Conforme a lo visto, el ámbito de aplicación

86. Una razón para actuar que tenga mayor peso que otra implica que esta prevalece o supera, pero no cancela. La diferencia entre superación y cancelación radica en la relevancia. La superación supone aplicabilidad de una razón, a pesar de la otra, a mérito de su peso en el caso en concreto. La cancelación supone la perdida de relevancia, debido a la ocurrencia de una de las condiciones para que ello ocurra.

87. Esto también se explica empleando la distinción entre aplicabilidad prima facie y aplicabilidad all things considered. 
de las normas es limitado mediante condicionales conjuntivos y los de excepción, los cuales se diferencian entre sí por los objetos a los que refieren, pero son estructuralmente idénticos.

Ambos condicionales implican la adición de nuevas propiedades en el antecedente a efectos de generar distinciones respecto a la acción o hecho que se pretende regular (o no regular), pero el condicional de excepción refiere a un tipo específico de hechos a mérito de alguna razón. ¿¿Cuáles son estas razones? Aquellas razones que podemos identificar a partir del análisis de las operaciones jurídicas, en el plano de la justificación de cada propiedad en el antecedente, en el estudio de los efectos que produce la distribución de la carga de la prueba o en la noción de razonabilidad de las consecuencias jurídicas de una norma, tal y como postulan quienes adoptar una aproximación conceptualista expuesta en el presente trabajo.

\section{CONCLUSIONES}

Las diversas teorizaciones sobre la noción de excepción tienen propósitos diferentes. Quienes adoptan una aproximación retórica proponen que uso del término «excepciones» forma parte de una argumentación pragmática, es decir, que tienen por objeto persuadir a la audiencia. Además de ello, pretenden negar que tengamos una diferencia lógica entre las diversas propiedades contenidas en el antecedente. Asumen esta postura, principalmente, por considerar que no es posible diferenciar entre una condición conjuntiva de aplicación y una de excepción. Quienes sostienen una aproximación conceptualista, en cambio, ofrecen tipos de análisis en los cuales «excepción» y «exceptuar» clarifican puntos relevantes sobre cómo están estructuradas las normas y resultados de operaciones que realizamos con normas.

Identificar qué propiedades operan como excepciones y cómo crearlas coadyuva mejorar nuestras prácticas reconstructivas de nuestras operaciones con normas, así como de sus resultados, a efectos de dar descripciones más precisas del derecho y como herramienta analítica a ser empleada por quienes pretendan diseñar excepciones en normas o exceptuar una norma en un proceso judicial.

Dicho valor se obtiene en mayor medida siempre que consideremos que la noción de excepción sea entendida a manera de verbo, «exceptuar», la cual da cuenta cómo modificamos la aplicabilidad interna de la norma, esto es, como la operación de introducir nuevas propiedades que reducen el alcance del caso genérico previsto en el antecedente. En efecto, entender la noción como sustantivo, «excepción», sola ofrece una aclaración de resultados contingentes (en función a como reconstruyamos la justificación de una norma o como se distribuya la carga de la prueba) de haber exceptuado la norma. 


\section{BIBLIOGRAFÍA}

Alchourrón, C,. 2010: Fundamentos para una Teoría General de los Deberes, Madrid, Marcial Pons.

Andrade, M., 2018: «Excepciones a derechos», Eunomía (N.o 14), 226-243.

Austin, J.L., 1975: Ensayos Filosóficos, Madrid: Revista de Occidente.

Austin, J.L., 1981: Sentido y percepción, Madrid: Tecnos.

BAYón, J.C., 2003: "¿Por qué es derrotable el razonamiento jurídico?» En: BAYón, J.C. y Rodríguez, J., Relevancia normativa en la justificación de las decisiones judiciales, Bogotá: Universidad Externado de Colombia, 263-205.

Bentham, J., 1970: On law in general, Londres: London Anthole Press.

Bouvier, H., 2012: Particularismo y derecho. Un abordaje pospositivsta en el ámbito práctico, Madrid: Marcial Pons.

Celano, B., 2009: “Defeasibility” y ponderación. Sobre la posibilidad de revisiones estables». En: Celano, B., Derecho, justicia, razones. Ensayos 2000-2007, Madrid: CEPC, 235-254.

Celano, B., 2017: «Particularismo, psicodeóntica. A propósito de la teoría de la justificación judicial de Manuel Atienza». En: Aguiló Regla, J. y Grandéz, P., Sobre el razonamiento judicial. Una discusión con Manuel Atienza, Lima: Palestra, 59-102.

Celano, B., 2012: «True Exceptions». En: Ferrer, J. y Ratti, G.B, The logic of legal requirements. Essays on defeasibility, Oxford: Oxford University Press, 268-287.

Celano, B. y Brigaglia, M., 2017: «Reasons, Rules, Exceptions: Towards a Psychological Account", Analisi e dirito, 131-144.

Duarte D'Almeida, L., 2015: Allowing for Exceptions. A theory of defenses and defeasibility in law, Oxford: Oxford University Press.

Fernández, M., 2004: Presunción de Inocencia y carga de la prueba en el proceso penal, leída ante la Comisión de la Universidad de Alicante. Disponible en:

https://rua.ua.es/dspace/bitstream/10045/11013/1/Fernandez-Lopez-Mercedes.pdf

Finkelstein, C. O., 200: «When the rule Swallows the exception», Penn Law: Legal Scholarship Repository (Vol. 19), 147-175.

Greenawalt, K., 1986: «Distinguishing justifications form excuses», Law and Contemporary Problems (N. 0 49), 89-108.

Hart, H.L.A., 1982: Essays on Bentham, Studies in jurisprudence and political theory, Oxford: Clarendon Press.

Hart, H. L. A., 2009: El Concepto de Derecho, Buenos Aires: Abeledo Perrot, 3ra Edición.

Hohfeld, W. N., 1991: Conceptos jurídicos fundamentales, Ciudad de México: Fontamara.

Honore, A., 1977: «Real Laws». En: Hacker, P.M.S. y Raz, J., Law, Morality and society. Essays in honour of H.L.A. Hart, Oxford: Clarendon Press, 99-118.

MacCormick, N., 1995: «Defeasibility in law and logic». En: Bankowski, Z., White, I. y HaHn, U., Informatics and the foundations of legal reasoning, Dordrecht: Springer.

MacCormick, N., 2016: Retórica y Estado de derecho, una teoría del razonamiento jurídico, Lima: Palestra.

Mendonca, D., 2012: «Exceptions». En: Ferrer, J. y Ratti, G.B, The logic of legal requirements. Essays on defeasibility, Oxford: Oxford University Press, 202-208.

Moreso, J.y Navarro, P., 1996: "Aplicabilidad y eficacia de las normas jurídicas», Isonomía (N. ${ }^{\circ}$ ), 119-139. 
Miller, L., 1956: «Rules and Exceptions», Ethics (Vol. 66, N.o 4), 262-270.

Prakken, H., 1997: Logical tools for modelling legal argument, Dordrecht: Springer.

Rodríguez, J., 2003: «LA DERrotabilidad de las normas JurídicAS». En: BAyón, J.C. y Rodríguez, J., Relevancia normativa en la justificación de las decisiones judiciales, Bogotá: Universidad Externado de Colombia, 67-102.

Rodríguez, J., 2006: «Un dilemma en la representación de normas condicionales», Isonomía (N. $\left.{ }^{\circ} 23\right)$, 97-114.

RaZ, J., 1986: El concepto de sistema jurídico, Ciudad de México: UNAM.

Schauer, F., 1991: "Exceptions», The University of Chicago Law Review (Vol. 58, N.o 3), 871-899.

Schauer, F., 2004: Las reglas en juego. Un examen filosófico de la toma de decisiones basada en reglas en el derecho y en la vida cotidiana, Madrid: Marcial Pons.

Stone, J., 1985: Precedent and law, Melbourne: Butterworths.

Susskind, R., 1987: Expert Systems in Law. A jurisprudential inquiri, Oxford: Clarendon Press. Williams, G., 1988: "The logic of "exceptions"», The Cambridge Law Journal (Vol. 47, N.o 2), 261-295.

Zorzetto, S., 2011: La norma speciale, una nozione ingannevole, Pisa: ETS

ZorzetTo, S., 2013: «Gerarchie normative e criteri di risoluzione delle antinomie. L'approccio realistico non ingenuo di Jordi Ferrer e Jorge Rodríguez», Analisi e Diritto, 69-94.

Zuleta, H., 2008: Norma y Justificación, Madrid, Marcial Pons. 